\title{
Blending of Cellulose and Chitosan in Alkyl Imidazolium Ionic Liquids
}

\author{
Olga Kuzmina, ${ }^{1}$ Thomas Heinze, ${ }^{2}$ and Dariusz Wawro ${ }^{1}$ \\ ${ }^{1}$ Fibres from Natural Polymers Department, Institute of Biopolymers and Chemical Fibers, Skłodowskiej-Curie 19/27, \\ 90-570 Lodz, Poland \\ ${ }^{2}$ Institut für Organische Chemie und Makromolekulare Chemie, Friedrich Schiller University of Jena, Humboldtsraße 10, \\ 07743 Jena, Germany
}

Correspondence should be addressed to Olga Kuzmina, olgakuzmina.chem@gmail.com

Received 12 September 2012; Accepted 11 October 2012

Academic Editors: A. Hashidzume and A. V. Popov

Copyright (๑) 2012 Olga Kuzmina et al. This is an open access article distributed under the Creative Commons Attribution License, which permits unrestricted use, distribution, and reproduction in any medium, provided the original work is properly cited.

The production of cellulose/chitosan blends in alkyl imidazolium ionic liquids (ILs) was studied in this work. Selected organic solvents, such as dimethyl sulfoxide, ethyl acetate, and diethyl ether, were used as cosolvents. The addition of cosolvents decreased the viscosity of cellulose/chitosan solutions in ILs and facilitated the dissolution of polysaccharides, thereby decreasing the $E_{\text {act }}$ and polymer aggregates sizes in the solutions. The cellulose/chitosan films were produced from the studied solutions. The presence of one of cosolvent and ILs in the blended films was confirmed by FTIR spectroscopy. The blended film is stronger than pure cellulose film, and the addition of cosolvents has an influence on its mechanical properties.

\section{Introduction}

Since 2002 ILs have been established as direct solvents for cellulose dissolution $[1,2]$. The advantages of using ILs for cellulose processing have been widely studied over the last decade $[1,3,4]$. Cellulose films and fibers produced from ILs solutions could be modified to improve their water absorption, dyeability, and mechanical and bactericidal properties [5-7]. It was found that ILs could dissolve not only cellulose but other carbohydrates [8], chitin, chitosan [9-11], and starch [12] proteins such as Bombyx mori silk fibroin $[13,14]$ and wool keratin [15]. It is possible to obtain unique mixtures of polymers in ILs, for example, mixtures of cellulose, starch, and lignin [16]. Binary blends of natural polymers such as cellulose and chitosan are promising systems for creating new polymer materials like blended films, fibers, and sponges. One of the main problems encountered with the blending of two polymers is its compatibility, which is dependent upon the formation of interand intramolecular interaction between the macromolecules. Compared to cellulose, the reactivity of chitosan is more versatile due to the presence of $\mathrm{NH}_{2}$ groups [17]. The blending of these two polymers could be a prospective way to their modification and improving the properties of the final products.

The similarity in the chemical structures of chitosan and cellulose predicts their compatibility and the possibility of their homogeneous blending. Cellulose, as most of the naturally occurring polysaccharides, is acidic in water media [18], whereas chitosan is a basic polysaccharide [19]. The research into cellulose-chitosan blending shows great potential for the materials that could be obtained from this method. The intramolecular interactions between molecules of both polymers were confirmed [19], and cellulose/chitosan films obtained with high content (up to $20 \mathrm{wt} \%$ ) of chitosan in real conditions are stronger than those calculated by the additive rule from the component's ratios [20]. Early are known works on blending of chitosan and cellulose in other solvents such as $\mathrm{NaOH} /$ thiourea, trifluoroacetic acid, N-methylmorpholine-N-oxide (NMMO), and under enzymatic treatment. The results were not entirely satisfying; the components were not very miscible, or deep depolymerisation of both polymers occurred. A preparation for a blend of chitosan and viscose rayon is known by regenerating $\mathrm{NaOH}$ solution of the xhantogenated blend of chitosan and dissolving pulp [21]. Microcrystalline chitosan 
(MCCh), which could be easily obtained from chitosan [22], is biocompatible, bioactive, nontoxic, and hydrophilic. MCCh was used previously to improve the properties of viscose fibers [23].

Xie et al. reported in 2006 [24] that the ionic liquid 1butyl-3-methylimidazolium chloride (BMIMCl) could dissolve up to $10 \mathrm{wt} \%$ of chitosan after 5 hours heating at $110^{\circ} \mathrm{C}$ under an inert atmosphere $\left(\mathrm{N}_{2}\right)$ with mechanical stirring. Recently, Wendler et al. (2010) reported that 1-ethyl3-methylimidazolium acetate (EMIMAc) cannot dissolve chitosan [25]. There are also a few recent patents describing methods of producing chitosan fibers [26] and films [27] from chitosan/IL solutions using ionic liquids, such as carboxyl alkyl imidazolium ILs, with cation alkyl imidazole and anion $\mathrm{Cl}^{-}, \mathrm{CH}_{2} \mathrm{ClCOO}^{-}$, and $\mathrm{CH}_{3} \mathrm{COO}^{-}$. The blending of cellulose with chitosan as a prospective method to modify cellulose materials has found interest in recent years. Some patents [28-30] describe the methods of producing cellulose/chitosan composite films, fibers, and foams using ILs, for example, alkyl imidazolium chloride/acetate and mixture of 1-H-3-methyl imidazolium chloride with other alkyl imidazolium chloride/acetate IL. There is also literature data available on composite biologically active fibers based on chitosan-coated Lyocell fibers [22]. However, some of these reports suggest the use of aqueous solutions of $\mathrm{NaOH}$ as a pretreatment for the chitosan $[22,26]$, which complicates the technological process and decreases the safety of its production. The successful blending of cellulose and chitosan in 1-butyl-3-methylimidazolium acetate was recently confirmed by Stefanescu et al. [11]. The obtained film possessed higher thermostability, but the proposed time of blending was several days at $85-95^{\circ} \mathrm{C}$, which is not sustainable for the technical implementation.

It is known that the addition of cosolvent may facilitate dissolution of polysaccharide in ILs [31, 32]. Different cosolvents were studied in order to improve the properties of cellulose solutions in ILs. Recently, Ma et al. reported the blending of cellulose and chitosan in a 1-butyl-3methylimidazolium chloride/glycine hydrochloride mixture [33]. The fibers obtained show higher tensile strength in comparison with regenerated cellulose fibers but reduced elongation at break.

The blended material could be obtained in both solid and liquid states. Solid-phase blending is provided under high pressure and shear deformation [23]. The blend material could be prepared from the solution by two methods: the dissolution of polymers separately in a common solvent followed by the combination of these two solutions or the blending of solid polymers and then the simultaneous dissolution of both polymers together in one solvent.

The aim of this work is to study the direct dissolution of chitosan and cellulose/chitosan blends in ILs and to determine the effect of selected cosolvents on the dissolution process in order to facilitate the process of producing blended materials for different purposes, including medical or textile applications, and to make this process more "green." We believe that the use of ILs as a solvent, replacing the previously used and more cumbersome solvents, will greatly benefit the technology. It was found that aprotic cosolvents allow for the dissolution of cellulose in ILs much faster and easier [32]. Few cosolvents are used in this work in order to make dissolution of polymers in ILs more efficient and to study the novel cellulose/chitosan/IL/cosolvent system. Blended films were produced from solutions as proof of successful polysaccharide blending. The mechanical properties of blended films were compared with the properties of cellulose films produced from the solution in ILs.

\section{Experimental}

2.1. Materials. High-purity wood-derived chemical cellulose pulp Alicell-Super (degree of polymerization, DP, 599), produced from western hemlock (Western Pulp Inc., Canada), was mechanically dispersed with Corner mill (calculated alpha content 93.5\%, moisture content 8\%). Chitosan "A" "Polymar Brazil" $\left(M_{w} 54,400 \mathrm{~g} / \mathrm{mol}\right.$, moisture content $9.70 \%$, deacetylation degree (DD) $76.90 \%$, ash content 3.10\%, and nitrogen content $6.90 \%$ ), chitosan "B" "Primex Chito-Clear FG 90" ( $M_{w}$ 121,500 g/mol, moisture content $10.75 \%$, DD $83.20 \%$, ash content $0.40 \%$, and nitrogen content $6.84 \%$ ), and microcrystalline chitosan "C" (MCCh) ( $M_{w} 341,800 \mathrm{~g} / \mathrm{mol}$, DD 83.2\%, moisture content $12.06 \%$, ash content $0.40 \%$, and nitrogen content $6.84 \%$ ) were purchased from IBWCh, Poland. 1Butyl-2,3-dimethylimidazolium chloride (BDMIMCl), 1butyl-3-methylimidazolium chloride (BMIMCl), 1-ethyl3-methylimidazolium acetate (EMIMAc), and 1-butyl-3methylimidazolium acetate (BMIMAc) were purchased from Sigma Aldrich, Germany. Dimethyl sulfoxide (DMSO), ethyl acetate (EAc), and diethyl ether (DEE) were obtained from $\mathrm{POCH}$, Poland.

2.2. Preparation of Blended Solutions in IL. The blends of cellulose and chitosan were obtained by 2 methods: simultaneous mixing of cellulose, chitosan, and ILs or by separate preparation of solution of each polymer in the same IL following subsequent combination. To separate preparations of the solutions of each polysaccharide, microwave irradiation 90 Wat during 5-120 s, acid media such as $10 \mathrm{wt} \% \mathrm{IL} /$ water solution, and different temperatures $\left(25,50,75\right.$, and $\left.110^{\circ} \mathrm{C}\right)$ over a period of $4 \mathrm{~h}$ with stirring were applied to dissolve chitosan. To the solution of chitosan in IL, the cellulose/IL solution was added in calculated proportions. The two solutions in a common solvent were stirred at different temperatures $\left(50-110^{\circ} \mathrm{C}\right)$ to obtain a homogeneous blend solution. In the second method, simultaneous dissolution of blended polysaccharides was carried out at different temperatures (room temperature: 50,75 , and $110^{\circ} \mathrm{C}$ ) over 4 hours with stirring. Cosolvents were added simultaneously with other components at the preparation step in amount of $5 \mathrm{wt} \%$. Turbidity, measured as an indicator of the progress of dissolution, was determined with an optical microscopy method.

2.3. Determination of $p H$ Values of IL/Water Solutions. $\mathrm{pH}$ measurements of IL/water solution with IL content 10, 20, and $50 \mathrm{wt} \%$ were recorded with a $\mathrm{pH}$ meter, Metler Toledo, Germany. 
2.4. Viscosity Measurements. Viscosity measurements were carried out with a Rheometer (Rheological Instruments Inc.) using the plate-plate system. Viscosity measurements were carried out with a shear rate in the range of gradient 5$120 \mathrm{~s}^{-1}$ (at $90^{\circ} \mathrm{C}$ ) and temperature heating from 0 to $100^{\circ} \mathrm{C}$ at constant shear rate.

\subsection{Preparation of Cellulose and Cellulose/Chitosan-Blended} Films. Cellulose and mixtures of cellulose/chitosan were dissolved in ILs and were casted onto a warm glass plate $\left(40^{\circ} \mathrm{C}\right)$, smoothed with a glass stick to obtain a thickness of $0.5 \mathrm{~mm}$, and rinsed with distilled water to remove solvent. The solvents utilized are miscible with water. Solvent removal was carried out in water in a tub, with at least 30 minutes of rinsing after each of 3 washes.

2.6. Mechanical Measurements of Blended Films. The tensile strength and elongation at break of the obtained films were measured with an Instron-5544 apparatus according to ISO 527-3:1998.

2.7. Thermogravimetric (TG) Analysis. TG analysis of samples cellulose, chitosan, and blended films was carried out by an NETZSCH STA 409 PC/PG differential scanning calorimeter at a heating rate of $10 \mathrm{~K} / \mathrm{min}$ under a continuous nitrogen flow in a temperature range between 20 and $500^{\circ} \mathrm{C}$.

2.8. Fourier Transform Infrared Spectroscopy (FTIR). Fourier transform infrared spectroscopy (FTIR) was made with a Genesis Series FTIRTM (Unicam Ltd.) spectrometer. The measurements were provided in the midinfrared region, approximately $4000-500 \mathrm{~cm}^{-1}$, using a $\mathrm{KBr}$ tablet.

2.9. Gel Permeation Chromatography (GPC). GPC was provided with a set of three PLgel Mixed A columns $(300 \times 7.5 \mathrm{~mm})$ with a guard column (Polymer Laboratories Ltd.). N,N-dimethylacetamide/lithium chloride (DMAC/0.5\% $\mathrm{LiCl}$ ) solution was used as an eluent. The cellulose component was extracted from blended films using an acetate buffer, which dissolved the chitosan. The $M_{w}$ of cellulose and chitosan was analyzed, and the cellulose DP was calculated.

2.10. Determination of the Size of the Aggregates in Blended Solutions. Zetasizer Nano (Malvern Instruments Ltd., UK) was used for determining the size of polymers aggregates in solution at $90^{\circ} \mathrm{C}$. The Zetasizer system determined the size by first measuring the Brownian motion of the particles in the sample using dynamic light scattering (DLS) and then interpreting a size from this using established theories. The size range measured by this apparatus is $2 \mathrm{~nm}-3 \mu \mathrm{m}$.

\section{Results and Discussion}

3.1. Preparation of Blends. A selection of different types of chitosan, including microcrystalline chitosan, were used in this work. Chitosan degrades at high temperatures; therefore, to dissolve chitosan in the ILs, the samples were treated with
TABLE 1: The $\mathrm{pH}$ values of the studied IL/water solutions.

$\mathrm{pH}$ values of IL/water solutions ILs content at

\begin{tabular}{lccc} 
IL & \multicolumn{3}{c}{$20^{\circ} \mathrm{C}, \mathrm{wt} \%$} \\
& 10 & 20 & 50 \\
\hline BMIMCl & 6.85 & 6.75 & 6.27 \\
BMIMAc & 5.30 & 5.48 & 6.54 \\
EMIMAc & 6.17 & 6.36 & 7.73 \\
\hline
\end{tabular}

microwave treatment first. Even at the lowest power setting available with our equipment, the appearance of smoke was observed after few seconds of treatment, indicating the degradation of the chitosan. All types of chitosan degraded under microwave irradiation in ILs. Thus, an alternative dissolution procedure was adopted.

Acidic media were used in order to study the possibility of dissolution of chitosan under mild conditions (without heating). Solutions of water/IL have different $\mathrm{pH}$ values dependent on the water content of the mixture. After measuring the $\mathrm{pH}$ of the IL/water solutions, the most acid solution was chosen for chitosan dissolution. Table 1 shows the $\mathrm{pH}$ values of some studied solutions are presented.

At room temperature chitosan could be more effectively dissolved in $10 \mathrm{wt} \%$ BMIMAc/water solution, because of its greater acidity compared with the other studied solutions. An amount of $1 \mathrm{wt} \%$ of chitosan A and B could be easily dissolved in BMIMAc/water solution, containing $10 \mathrm{wt} \%$ of IL, over $1.5 \mathrm{~h}$ with magnetic stirring. Due the fact that chitosan $\mathrm{C}$ has a larger $M_{w}$ than the other types of chitosan, its dissolution takes much longer time. The concentration of $1 \mathrm{wt} \%$ of chitosan $\mathrm{C}$ in $10 \mathrm{wt} \%$ BMIMAc/water solution could be reached in $2.5 \mathrm{~h}$ at $25^{\circ} \mathrm{C}$. The water content of the obtained chitosan/IL/water solution was approximately $90 \mathrm{wt} \%$. This value was too high for mixing it with cellulose/IL solution; an excess of water would cause cellulose precipitation. Therefore, the water should be removed from the chitosan/IL/water system before mixing with the cellulose solution.

As it was discussed earlier, chitosan degraded rapidly at high temperatures; therefore, water was removed at low temperatures under vacuum conditions. The residual chitosan/IL system after the removal of water appeared to be a gelatinous material. The appropriate amount of cellulose/IL solution was added to chitosan/IL system after calculation of the component ratio in the obtained chitosan/IL system. The sample was heated to $50^{\circ} \mathrm{C}$ to blend the solutions of the two polysaccharides avoiding the degradation of chitosan. However, after heating over the course of 24 hours at $50^{\circ} \mathrm{C}$, and even at $110^{\circ} \mathrm{C}$, the blending of the components was ineffective; the system formed a heterophase gelatinous material. The formation of such a system is likely to occur due to the formation of a strong interaction between chitosan and IL on the removal of water from the system. The newly formed interaction between the two phases was so strong that the obtained chitosan/IL material was not miscible with the cellulose/IL solution, and it was insoluble in pure IL.

Among the ILs studied only EMIMAc and BMIMAc were able to dissolve at least $1 \mathrm{wt} \%$ of each of the analyzed 
chitosans; clear solutions were obtained after heating at $110^{\circ} \mathrm{C}$ for 4 hours. The unsuccessful experiments of the dissolution of chitosan in EMIMAc in previous studies [25] could be explained by the different parameters used during the dissolution procedure and also the different types of chitosan used. Out of the ILs studied, BMIMAc demonstrated the best dissolving ability toward chitosan. The most concentrated cellulose solutions could be also produced with acetate ILs, especially BMIMAc. A calculated amount of cellulose solution should be mixed with the chitosan solution to produce its blend. Cellulose solutions were obtained after heating at $110^{\circ} \mathrm{C}$ for 2 hours as described previously [34]. The mixture of cellulose and chitosan in ILs was stirred at $110^{\circ} \mathrm{C}$ for 30 minutes. The obtained blends presented clear, viscous solutions with polymer concentrations of up to $4.5 \mathrm{wt} \%$ cellulose and $0.5 \mathrm{wt} \%$ chitosan. The method of producing blends by heating could be facilitated by using the second method of blend preparation, by simultaneous dissolution of polymers in ILs. A selection of cellulose/chitosan blends were prepared in EMIMAc and BMIMAc at a temperature of $110^{\circ} \mathrm{C} .5 \mathrm{wt} \%$ of cellulose/chitosan polymer content could be easily obtained in IL after heating at $110^{\circ} \mathrm{C}$ for $4 \mathrm{~h}$.

Direct dissolution of chitosan in IL is very challenging; therefore, the progress of the dissolution was only observed in solutions with little chitosan content. The maximum ratio of the components of the cellulose/chitosan/IL systems achieved was $4.5 / 0.5 / 95$, independent of the chitosan type. The ratio of polysaccharides was $90 \mathrm{wt} \%$ cellulose to $10 \mathrm{wt} \%$ chitosan.

3.2. Influence of Cosolvents on Properties of Blends. Aprotic solvents are efficient cosolvents for cellulose dissolution in ILs $[32,35]$. DMSO, DEE, and EAc were chosen for cellulose/chitosan solutions in order to facilitate the dissolution process and to improve the rheological properties of the studied solutions. In Figure 1 the dependence of shear rate on the viscosity of the cellulose/chitosan solution in BMIMAc with cosolvents is shown. The example given is for cellulose/chitosan C blend; however, other chitosan powders show the same behavior. It was found that cellulose/chitosan blends in ILs have the highest viscosity, and the addition of cosolvents decreases its viscosity. The same dependence was found for cellulose solutions in ILs. Cellulose/chitosan blends, both in EMIMAc and BMIMAc, have very similar viscosity values. The decrease in viscosity values is not in the line with mixing rule for $\mathrm{IL} /$ cosolvent mixture. The viscosities of the pure cosolvents decrease in the order DMSO $>$ EAc > DEE; however, the viscosity of the blends decreases independently of used cosolvents decreases in order EAc $>$ DMSO > DEE. The reason of these changes could be the interaction of cosolvents with components of blends, especially with chitosan, which is more reactive due to presence of $\mathrm{NH}_{2}$ groups.

The temperature dependence of viscosity was studied for cellulose/IL and cellulose/chitosan/IL solutions over a temperature range of $273-373 \mathrm{~K}$. Figure 2 shows an example of the influence of temperature on viscosity for cellulose/EMIMAc and cellulose/chitosan B/EMIMAc solutions with and without the addition of cosolvents. The example

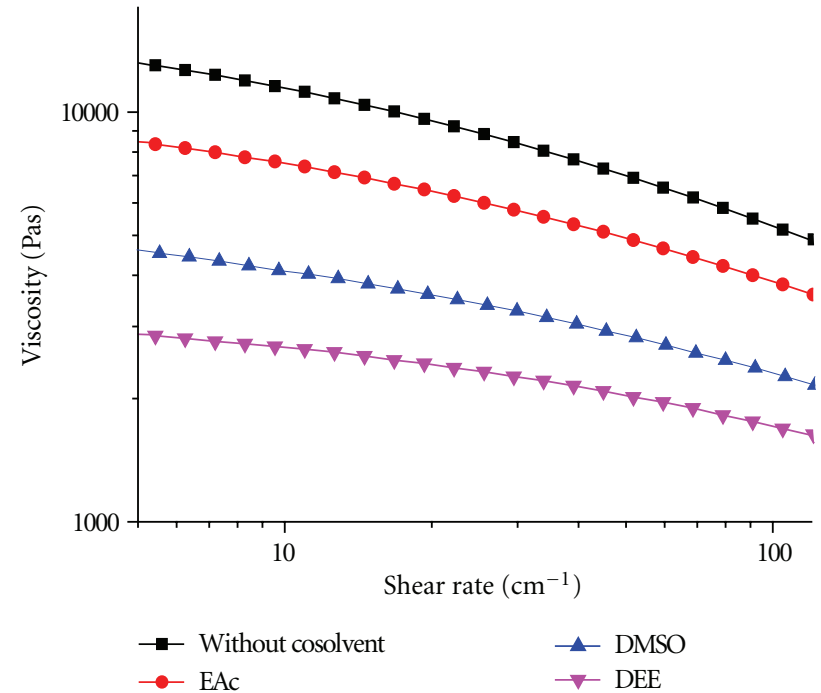

FIGURE 1: The influence of the shear rate on the viscosity of cellulose/chitosan C solution in BMIMAc with and without cosolvents at $90^{\circ} \mathrm{C}$. The ratio of components cellulose: chitosan:BMIMAc: cosolvent is $4.5: 0.5: 90: 5$.

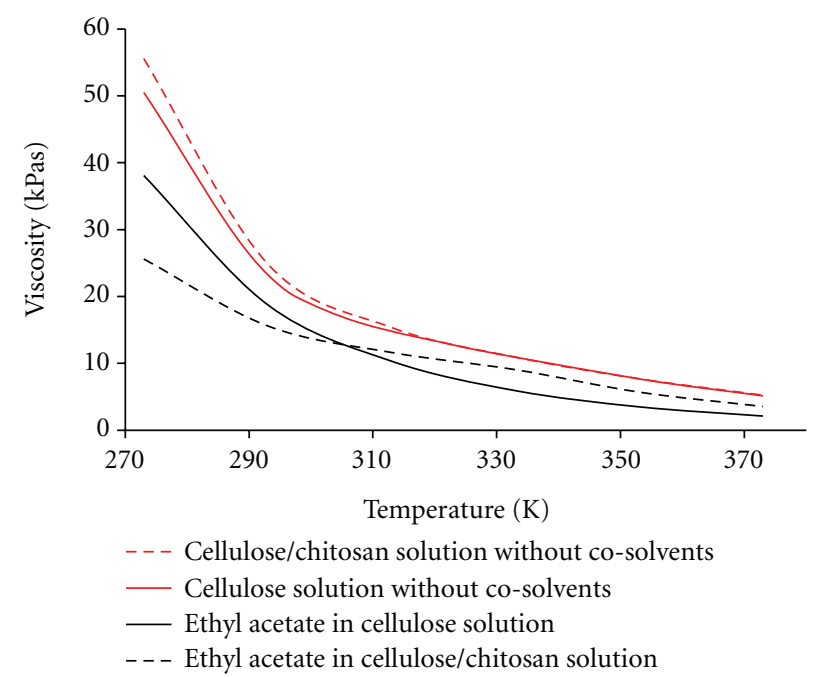

FIGURE 2: The temperature dependence of viscosity of $5 \mathrm{wt} \%$ cellulose solutions in EMIMAc with and without cosolvents (solid lines) and $4.5 \mathrm{wt} \%$ cellulose $/ 0.5 \mathrm{wt} \%$ chitosan A solutions in EMIMAc with and without cosolvents (dashed lines) on temperature at constant shear rate $10 \mathrm{~s}^{-1}$. The concentration of cosolvents was $5 \mathrm{wt} \%$.

given is for EAc cosolvent. Cellulose/chitosan solutions are more viscous than pure $5 \mathrm{wt} \%$ cellulose solution due to additional interactions between the two polysaccharides and poorer chitosan dissolution in ILs. However, upon the addition of EAc the viscosity of the blend decreased dramatically and became lower than for pure cellulose solution. This is probably due to the destruction of the cellulose and chitosan structures and intermolecular interactions formed upon swelling with EAc and also the extensive evaporation upon heating.

The Arrhenius plot for calculating the activation energy $\left(E_{\text {act }}\right)$ from the temperature dependence of viscosity of 


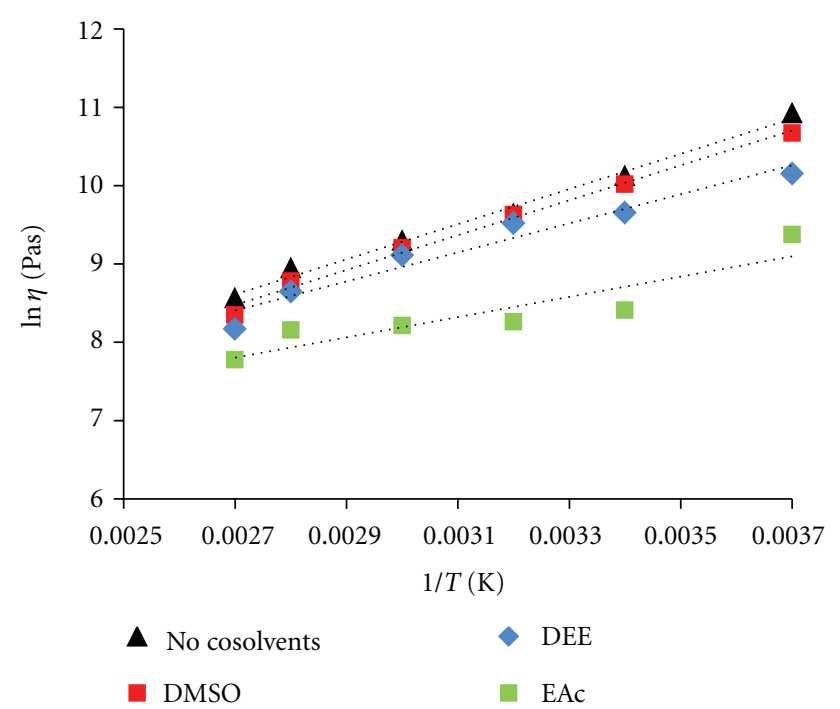

FIgURE 3: Arrhenius plot for cellulose/chitosan A blend solution in EMIMAc with cosolvents. Trend lines correspond to first Arrhenius approximation.

cellulose/chitosan solution with and without cosolvents was plotted (Figure 3). Despite the fact that the plotted dependences were not exactly linear, according to the literature $[32,36]$, the activation energies were calculated as a first approximation. The plot of $\ln k$ versus $1 / T$ at right gives a credible straight line as expected since the Arrhenius expression for the $T$ dependence of rate constants is

$$
k=A e^{-E_{\text {act }} / R T}
$$

By taking the log of both sides and rearranging we can get the equation of a straight line $(y=a x+b)$ :

$$
\ln k=\left(-\frac{E_{\mathrm{act}}}{R}\right)\left(\frac{1}{T}\right)+\ln A,
$$

meaning that the slope is $-E_{\text {act }} / R$ or $E_{\text {act }}=-R \times$ (slope) $=-8.314 \mathrm{~J} / \mathrm{mol} K \times$ (slope). The calculated $E_{\text {act }}$ for the cellulose/IL and cellulose/chitosan/IL solutions was presented in Table 2. The standard error for these calculations is $5 \%$. All cellulose/chitosan solutions in ILs had concentrations as presented in Figure 3 (4.5:0.5:90:5 for cellulose: chitosan:BMIMAc: cosolvent solutions and 4.5 : $0.5: 95$ for cellulose : chitosan : BMIMAc, resp.).

The larger the value of $E_{\text {act }}$, the harder the dissolution of polymers in ILs. $E_{\text {act }}$ increases with polymer concentration [32]. Cosolvents added to cellulose/chitosan solutions at $5 \mathrm{wt} \%$ concentration decrease its $E_{\text {act }}$ (Table 2 ) and cause the values to be similar for the cellulose solutions.

Thus, the dissolution of polysaccharides starts easily in the presence of cosolvents due to penetration of cosolvents into the polysaccharide's structure and subsequent replacement of cosolvent by IL. This mechanism facilitates the dissolution of polysaccharides in ILs. In Table 2 it is shown that the sizes of polymer aggregates were found to be larger in EMIMAc solutions than in BMIMAc solutions. The polymer aggregates sizes in cellulose solutions are smaller

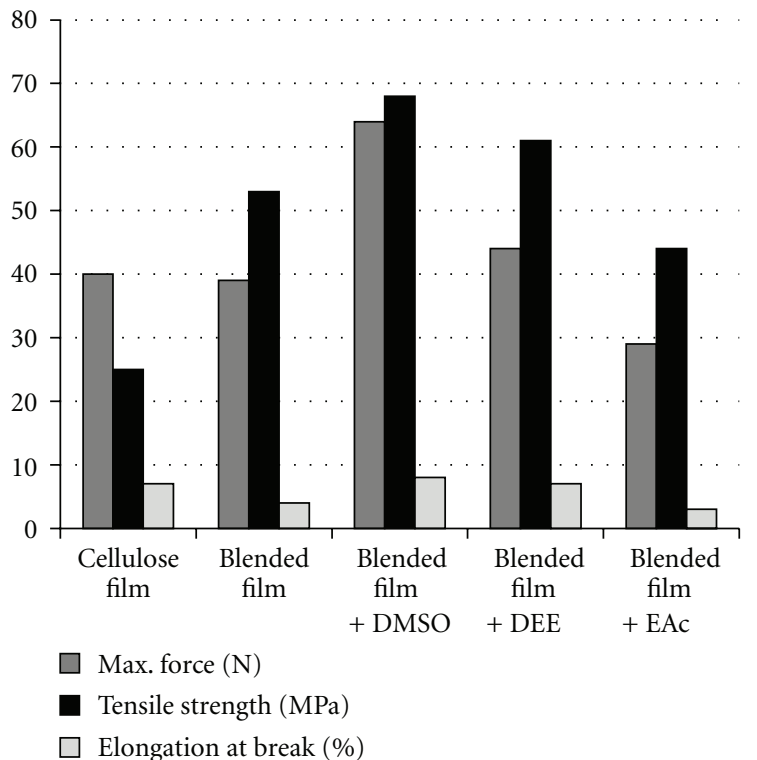

Figure 4: Comparison of mechanical properties of pure cellulose film, obtained from $5 \mathrm{wt} \%$ cellulose/EMIMAc solution, cellulose/chitosan B film obtained without cosolvents from $4.5 \mathrm{wt} \%$ cellulose $/ 0.5 \mathrm{wt} \%$ chitosan $/ 90 \mathrm{wt} \%$ EMIMAc solution, and cellulose/chitosan B films, obtained from $4.5 \mathrm{wt} \%$ cellulose $/ 0.5 \mathrm{wt} \%$ chitosan $/ 90 \mathrm{wt} \%$ EMIMAc/5 wt $\%$ cosolvent solutions.

than in cellulose/chitosan blends. The reason for the increase in size of aggregates in blended solution could arise from the aggregation of chitosan, which is least soluble, and aggregation of cellulose and chitosan molecules caused by the compatibility of these polymers. The addition of cosolvents decreases the size of polymer aggregations in both IL solutions, which means the cellulose and chitosan molecules are better dissolved in the presence of cosolvents, and the solutions with these cosolvents are more homogeneous.

\subsection{Cellulose/Chitosan Films}

3.3.1. Mechanical Characteristics of Blended Films. The mechanical characteristics of cellulose films were compared with those of the blended films obtained with and without the use of cosolvents. The blended film had a relatively similar maximum force and elongation at break in comparison with cellulose film, but the blended film had better tensile strength (Figure 4).

The addition of DMSO and DEE improved the mechanical characteristics of the blended films, but the addition of EAc slightly decreased its values; the cosolvents used may act as plasticizers. The dissolution of the polymer blends in the presence of EAc is very intensive, and the molecular structure of polysaccharides was greatly destroyed, which leads to inferiority in the mechanical properties of the blended films. Generally, the blended films were stronger than the pure cellulose films, produced from the ILs.

3.3.2. Degradation of Cellulose and Chitosan during Preparation of Blended Films. The influence of blending polysaccharides on the degradation of cellulose was studied by means 
TABLE 2: The sizes of polymer aggregates (hydrodynamic radius) and $E_{\text {act }}$ of the cellulose/chitosan B solutions with cosolvents in comparison to cellulose solutions in ILs.

\begin{tabular}{|c|c|c|c|c|c|c|c|}
\hline \multirow{2}{*}{ IL } & \multirow{2}{*}{ Cosolvent } & \multicolumn{4}{|c|}{ Concentrations of components in the solutions, wt $\%$} & \multirow{2}{*}{$E_{\text {act }}, \mathrm{kJ} / \mathrm{mol}$} & \multirow{2}{*}{ Aggregates size, $\mathrm{nm}$} \\
\hline & & Cellulose & Chitosan & IL & Co-solvent & & \\
\hline \multirow{5}{*}{ EMIMAc } & - & 5 & 0 & 95 & 0 & 23 & 419 \\
\hline & - & 4.5 & 0.5 & 95 & 0 & 24 & 600 \\
\hline & DMSO & 4.5 & 0.5 & 90 & 5 & 21 & 552 \\
\hline & DEE & 4.5 & 0.5 & 90 & 5 & 21 & 203 \\
\hline & EAc & 4.5 & 0.5 & 90 & 5 & 19 & 368 \\
\hline \multirow{5}{*}{ BMIMAc } & - & 5 & 0 & 95 & 0 & 24 & 197 \\
\hline & - & 4.5 & 0.5 & 95 & 0 & 27 & 208 \\
\hline & DMSO & 4.5 & 0.5 & 90 & 5 & 25 & 199 \\
\hline & DEE & 4.5 & 0.5 & 90 & 5 & 21 & 196 \\
\hline & EAc & 4.5 & 0.5 & 90 & 5 & 20 & 175 \\
\hline
\end{tabular}

of GPC. The amount of chitosan extracted from blended film was approximately $3 \mathrm{wt} \%$. From the analysis of the extracted cellulose component it was found that the increase in chitosan content toward cellulose leads to a decreasing of the cellulose DP in the blended films (Table 3), which could lead to inferior mechanical properties of the produced films. We assumed that highly degradable chitosan promotes cellulose degradation. The molecular weight of chitosan decreases dramatically in solution with ILs, which is caused by temperature effect during the 4-hour dissolution process. Upon blending with cellulose in IL, chitosan degrades further despite cosolvents being unable to inhibit the chitosan degradation (Table 4).

We found that using a cosolvent to facilitate the dissolution of polysaccharides in ILs leads to additional swelling and deep destruction of the structure of chitosan, which resulted in lowering its $M_{w}$.

3.3.3. DTG Analysis of Blended Films. Using DTG analysis two peaks were observed for the degradation of cellulose/chitosan-blended film (Figure 5): the first peak corresponds to the degradation of chitosan and the second to cellulose degradation. The $\mathrm{OH}$-degradation occurs in larger rate; the peak at the beginning of heating before $100^{\circ} \mathrm{C}$ is much wider for blended film than that of the original polysaccharides. It could easily be explained by higher moisture content $(\sim 12 \%)$ of blended film. Generally, the blended film demonstrated higher thermostability than the original polysaccharides. This phenomenon could be explained by strong interactions between the polysaccharides in the blend, resulting in higher temperatures of degradation.

3.3.4. FTIR Spectroscopy of Blended Films. Cellulose/chitosan-blended films were produced as final products from the studied solutions. The blended films produced, with polymer concentrations of $4.5 \mathrm{wt} \%$ and $0.5 \mathrm{wt} \%$, respectively, consist theoretically of $90 \mathrm{wt} \%$ of cellulose and $10 \mathrm{wt} \%$ of chitosan. By the FTIR analysis it is possible to confirm the presence of chitosan in the blended films. Bands of $\mathrm{OH}-$ stretching $\left(3650-3200 \mathrm{~cm}^{-1}, 1200-1000 \mathrm{~cm}^{-1}\right)$ and $\mathrm{C}-\mathrm{H}$ stretching (2900-2850 $\left.\mathrm{cm}^{-1}, 1500-1300 \mathrm{~cm}^{-1}\right)$ frequencies,

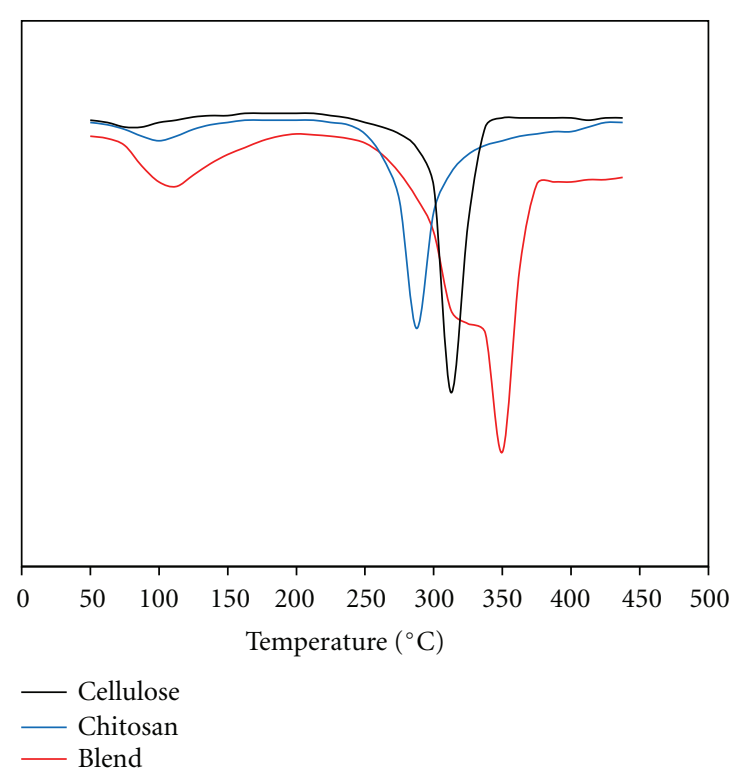

FIGURE 5: DTG plot of cellulose, chitosan A, and cellulose/chitosan blended film, obtained from BMIMAc solution.

which could be attributed to both polymers, were found in FTIR spectra (Figure 6) of the blended films.

Also, the peaks corresponded to $\mathrm{NH}$-stretch of amide and carbonyl group $\left(1597 \mathrm{~cm}^{-1}, 1659 \mathrm{~cm}^{-1}\right)$ of chitosan [31]; strong stretches of double bonding $\mathrm{C}=\mathrm{O}$ between 1600 and $1700 \mathrm{~cm}^{-1}$ and stretching of carboxyl group $\mathrm{COO}^{-}$at $\sim 1400$ and $1600 \mathrm{~cm}^{-1}$ were observed. The stretching of $\mathrm{C}-\mathrm{H}$ bonding $\left(\mathrm{CH}, \mathrm{CH}_{2}, \mathrm{CH}_{3}\right)$ is noticed around $1400 \mathrm{~cm}^{-1}$. There should not be much absorption in the $1700-1500 \mathrm{~cm}^{-1}$ region in a spectrum of pure cellulose because cellulose on its own has only a small band at $\sim 1630 \mathrm{~cm}^{-1}$ due to the $\mathrm{OH}$ deformation. The spectra of cellulose, obtained from IL solution, fulfill this condition (Figure 6). All peaks corresponded to $\mathrm{O}-\mathrm{H}, \mathrm{C}-\mathrm{H}, \mathrm{C}-\mathrm{O}$ but not the sugar ring bands between 1200 and $900 \mathrm{~cm}^{-1}$ were much more intensive for blended film than for pure cellulose.

At more close look at the chitosan spectra (Figure 7) few shifts of carbonyl group stretching $\left(1652 \rightarrow 1638 \mathrm{~cm}^{-1}\right)$ 
TABLE 3: The influence of the amount of chitosan A in blended film on the $M_{w}$ and DP of its cellulose component degradation.

\begin{tabular}{lcccc}
\hline \multicolumn{2}{c}{ Components ratio in solutions for films producing } & \multicolumn{2}{c}{ Analysis of cellulose component extracted from blended films } \\
Cellulose & IL & Chitosan & $M_{w}$, g/mol & DP \\
\hline 4.5 & 85 & 0.5 & 64,681 & 399 \\
4.9 & 85 & 0.1 & 69,995 & 432 \\
\hline
\end{tabular}

TABLE 4: $M_{w}$ of chitosan A contained in blended films obtained from cellulose/chitosan/IL and cellulose/chitosan/IL/cosolvent systems.

\begin{tabular}{lc}
\hline Chitosan & $M_{w}, \mathrm{~g} / \mathrm{mol}$ \\
\hline Original chitosan powder & 54,400 \\
Chitosan obtained from films prepared from the following: & 12,400 \\
Chitosan/EMIMAc $(0.5 \mathrm{wt} \%$ of chitosan $)$ solution & 7,600 \\
$4.5 \mathrm{wt} \%$ cellulose $/ 0.5 \mathrm{wt} \%$ chitosan/EMIMAc solution & 7,400 \\
$4.5 \mathrm{wt} \%$ cellulose $/ 0.5 \mathrm{wt} \%$ chitosan/EMIMAc/5 wt $\%$ DMSO solution & 6,900 \\
$4.5 \mathrm{wt} \%$ cellulose $/ 0.5 \mathrm{wt} \%$ chitosan/EMIMAc/5 wt $\%$ DEE solution
\end{tabular}

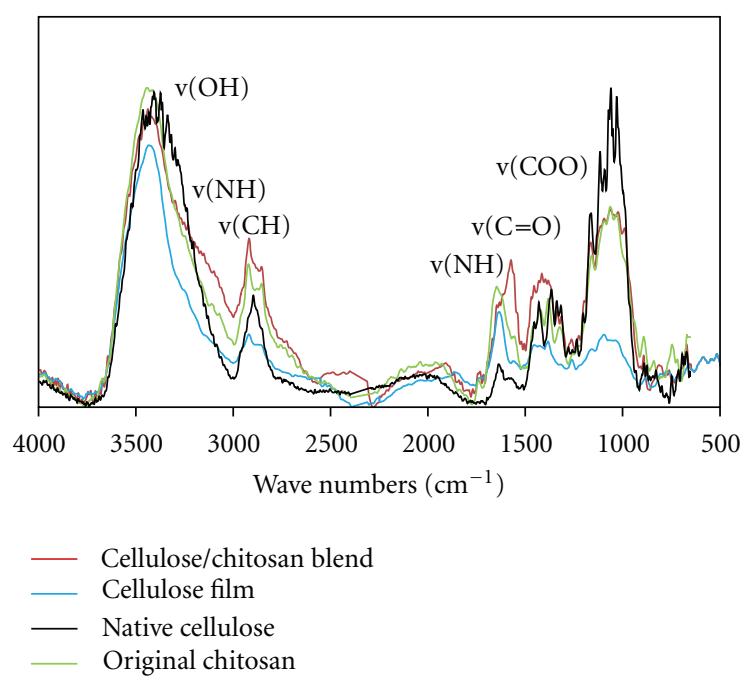

FIGURE 6: Comparison of FTIR spectra of films produced from EMIMAc solutions and original polysaccharides.

and amide group stretching $\left(1597 \rightarrow 1589 \mathrm{~cm}^{-1}\right)$ were found. At the same time, the stretching of $-\mathrm{NH}$ group is no longer observed in the spectra of blended film, because of its shifting to higher frequency and overlapping by stretching of carbonyl group. The shifting of amino and carboxyl group vibrations suggests that a reaction took place between the polysaccharides [11]. In contrast with previous studies of blending cellulose and chitosan in other solvents [11] there is no need to use additional reagents, for example, carboxymethylated chitosan, to improve the miscibility of polysaccharides in ILs.

\section{Conclusions}

Among the ILs studied, EMIMAc and BMIMAc are suitable to produce cellulose/chitosan blends by heating from all types of chitosan used in this study. Microwave treatment was not sufficient for chitosan dissolution and led to its rapid

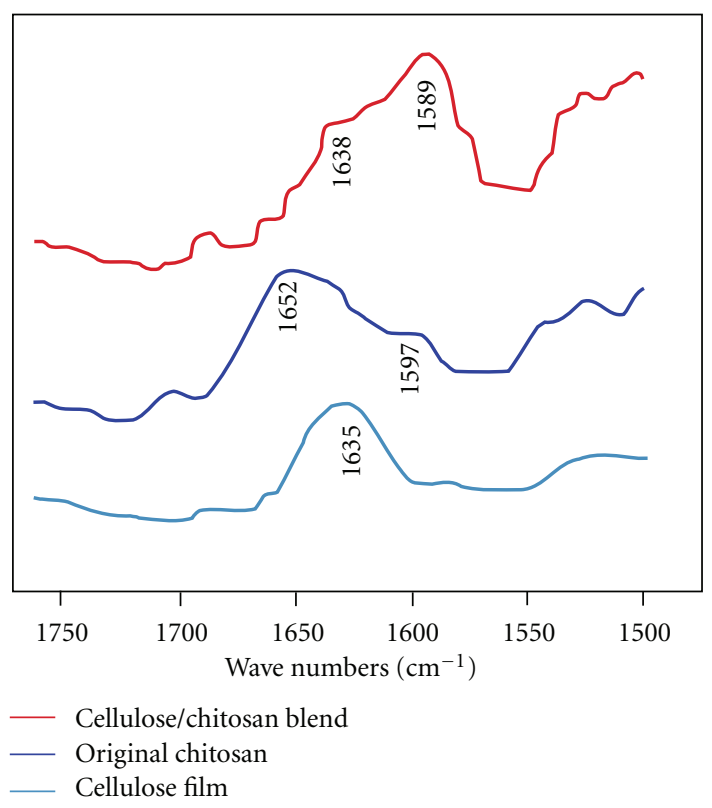

Figure 7: Shifting of characteristic peaks in cellulose/chitosan blend.

degradation. Simultaneous blending of components was more efficient than mixing the separately prepared chitosan and cellulose solutions in a common solvent. The addition of cosolvents decreased the viscosity of cellulose/chitosan solutions in ILs and facilitated the dissolution of polysaccharides. The use of cosolvents facilitates polysaccharides blending in ILs. Increasing the chitosan concentration in the blends promoted the degradation of the cellulose component. The addition of DMSO and DEE improved the mechanical properties of blended films. Despite different characteristics, all of three types of chitosan showed very similar dissolution and blending properties. Chitosan is a promising polysaccharide for cellulose modification. ILs are prospective and efficient solvents for blending cellulose and chitosan, which do not require chitosan derivatives to improve the miscibility of chitosan and cellulose. 


\section{Acknowledgments}

The research presented received funding from the European Community's Seventh Framework Program [FP7/20072013] under Grant Agreement no. PITNGA-2008-214015.

\section{References}

[1] R. P. Swatloski, S. K. Spear, J. D. Holbrey, and R. D. Rogers, "Dissolution of cellose with ionic liquids," Journal of the American Chemical Society, vol. 124, no. 18, pp. 4974-4975, 2002.

[2] R. Swatloski, R. Rogers, and J. Holbrey, "Dissolution and processing of cellulose using ionic liquids," US, 6824599, 2003.

[3] K. Massonne, "Alternative cellulose-manufacturing, forming, properties," in Proceedings of the 7th International Symposium, Rudolstadt, Germany, September 2006.

[4] S. Keskin, D. Kayrak-Talay, U. Akman, and Ö. Hortaçsu, "A review of ionic liquids towards supercritical fluid applications," Journal of Supercritical Fluids, vol. 43, no. 1, pp. 150180, 2007.

[5] S. Chao-Ming, S. Yeong-Tarng, and T. Yawo-Kuo, "Preparation and characterization of cellulose/chitosan blend films," Carbohydrate Polymers, vol. 78, no. 1, pp. 169-174, 2009.

[6] G. Yunlin, L. Xiaofei, Z. Yingping, and Y. Kangde, "Study of phase behavior on chitosan/viscose rayon blend film," Journal of Applied Polymer Science, vol. 67, no. 12, pp. 1965-1972, 1998.

[7] M. H. Struszczyk, F. Loth, and M. G. Peter, "Preparation of paper sheets containing microcrystalline chitosan," Advances in Chitin Science, vol. 4, pp. 128-135, 2000.

[8] O. A. El Seoud, A. Koschella, L. C. Fidale, S. Dorn, and T. Heinze, "Applications of ionic liquids in carbohydrate chemistry: a window of opportunities," Biomacromolecules, vol. 8, no. 9, pp. 2629-2647, 2007.

[9] S. Xiaoqi, B. Peng, J. Yang, J. Chen, and L. Deqian, "Chitosan(chitin)/cellulose composite biosorbents prepared using ionic liquid for heavy metal ions adsorption," AIChE Journal, vol. 55, no. 8, pp. 2062-2069, 2009.

[10] W. Xiao, Q. Chen, Y. Wu, T. Wu, and L. Dai, "Dissolution and blending of chitosan using 1,3-dimethylimidazolium chloride and 1-H-3-methylimidazolium chloride binary ionic liquid solvent," Carbohydrate Polymers, vol. 83, no. 1, pp. 233-238, 2011.

[11] S. Stefanescu, H. D. Daly, and I. I. Negulescu, "Biocomposite films prepared from ionic liquid solutions of chitosan and cellulose," Carbohydrate Polymers, vol. 87, pp. 435-443, 2012.

[12] K. Wilpiszewska and T. Spychaj, "Ionic liquids: media for starch dissolution, plasticization and modification," Carbohydrate Polymers, vol. 86, no. 2, pp. 424-428, 2011.

[13] D. M. Phillips, L. F. Drummy, R. R. Naik et al., "Regenerated silk fiber wet spinning from an ionic liquid solution," Journal of Materials Chemistry, vol. 15, no. 39, pp. 4206-4208, 2005.

[14] O. G. Kuzmina, E. S. Sashina, N. P. Novoselov, and M. Zaborski, "Blends of cellulose and silk fibroin in 1-buthyl3-methylimidazolium chloride-based solutions," Fibres and Textiles in Eastern Europe, vol. 77, no. 6, pp. 36-39, 2009.

[15] N. Hameed and Q. Guo, "Blend films of natural wool and cellulose prepared from an ionic liquid," Cellulose, vol. 17, no. 4, pp. 803-813, 2010.

[16] R. L. Wu, X. L. Wang, F. Li, H. Z. Li, and Y. Z. Wang, "Green composite films prepared from cellulose, starch and lignin in room-temperature ionic liquid," Bioresource Technology, vol. 100, no. 9, pp. 2569-2574, 2009.

[17] P. K. Dutta, J. Duta, and V. S. Tripathi, "Chitin and Chitosan: chemistry, properties and applications," Journal of Scientific and Industrial Research, vol. 63, no. 1, pp. 20-31, 2004.

[18] P. Nousiainen, M. I. Vehvila, H. Struszczyk, and E. J. Makinen, "Functional hybrid fibers of cellulose/microcrystalline Chitosan. I. Manufacture of viscose/microcrystalline chitosan fibres," Applied Polymer Science, vol. 76, pp. 1725-1730, 2000.

[19] M. Holmberg, J. Berg, S. Stemme, L. Ödberg, J. Rasmusson, and P. Claesson, "Surface force studies of Langmuir-Blodgett cellulose films," Journal of Colloid and Interface Science, vol. 186, no. 2, pp. 369-381, 1997.

[20] M. Hasegawa, A. Isogai, S. Kuga, and F. Onabe, "Preparation of cellulose-chitosan blend film using chloral/dimethylformamide," Polymer, vol. 35, no. 5, pp. 983-987, 1994.

[21] H. Struszczyk and P. Nousiainen, "Some aspects of viscose fibres modification by microcrystalline Chitosan," in Cellulose Sources and Exploitation, J. F. Kennedy, G. O. Phillips, and P. A. Williams, Eds., chapter 23, Ellias Horwood, New York, NY, USA, 1990.

[22] S. Janjic, M. Kostic, V. Vucinic et al., "Biologically active fibers based on chitosan-coated lyocell fibers," Carbohydrate Polymers, vol. 78, no. 2, pp. 240-246, 2009.

[23] S. Z. Rogovina and G. A. Vikhoreva, "Polysaccharide-based polymer blends: methods of their production," Glycoconjugate Journal, vol. 23, no. 7-8, pp. 611-618, 2006.

[24] H. Xie, S. Zhang, and S. Li, "Chitin and chitosan dissolved in ionic liquids as reversible sorbents of $\mathrm{CO}_{2}$," Green Chemistry, vol. 8, no. 7, pp. 630-633, 2006.

[25] F. Wendler, F. Meister, D. Wawro et al., "Polysaccharide blend fibres formed from $\mathrm{NaOH}, \mathrm{N}$-methylmorpholine- $\mathrm{N}$-oxide and 1-Ethyl-3-methylimidazolium acetate," Fibres and Textiles in Eastern Europe, vol. 79, no. 2, pp. 21-30, 2010.

[26] S. Yu, F. Liu, L. Li, C. Xie, S. Liang, and H. Ji, "A method to prepare chitosan fibre," CN Patent 10150353 0A, 20090812, 2009.

[27] Y. Xiong, Z. Wang, R. Wang, C. Wu, and H. Wang, "The preparation of chitosan/ionic liquid condustive composite film," CN Patent 10155061 0A, 20091007, 2009.

[28] F. Pang, J. Liu, J. Ma, M. Jiang, L. Li, and Y. Wu, "Preparation of regenerated cellulose/chitin composite material and prepared composite fiber," CN Patent 101372764 A, 20090225, 2009.

[29] L. Dai, W. Xiao, T. Wu, and W. Luo, "Dissolution and blending of chitosan using 1, 3-dimethyimidazolium chloride and 1H-3-methylimidazolium chloride binary ionic liquid solvent," CN Patent 101899171 A, 20101201, 2010.

[30] X. Li, Y. Sun, Q. Luo, J. Xu, and Y. Lu, "Method for preparing core-sheath composite fibers from chitosan and cellulose," CN Patent 101768790 A, 20100707, 2010.

[31] M. Luo, A. N. Neogi, and H. West, "Dissolution of cellulose in mixed solvent systems,” US 7828 936, 2009.

[32] M. Gericke, T. Liebert, O. A. E. Seoud, and T. Heinze, “Tailored media for homogeneous cellulose chemistry: ionic liquid/cosolvent mixtures," Macromolecular Materials and Engineering, vol. 296, no. 6, pp. 483-493, 2011.

[33] B. Ma, M. Zhang, C. He, and J. Sun, "New binary ionic liquid system for the preparation of chitosan/cellulosecomposite fibers," Carbohydrate Polymers, vol. 88, pp. 347-351, 2012.

[34] O. Kuzmina, E. Sashina, S. Troshenkowa, and D. Wawro, "Dissolved state of cellulose in ionic liquids - the impact of water," Fibres and Textiles in Eastern Europe, vol. 80, no. 3, pp. 32-37, 2010. 
[35] N. P. Novoselov, O. G. Kuzmina, and S. V. Trochenkova, "Ionic liquids as a new solvents for natural polymers," Chemical Fibers, vol. 3, pp. 75-79, 2008.

[36] R. Sescousse, K. A. Le, M. E. Ries, and T. Budtova, "Viscosity of cellulose-imidazolium-based ionic liquid solutions," Journal of Physical Chemistry B, vol. 114, no. 21, pp. 7222-7228, 2010. 

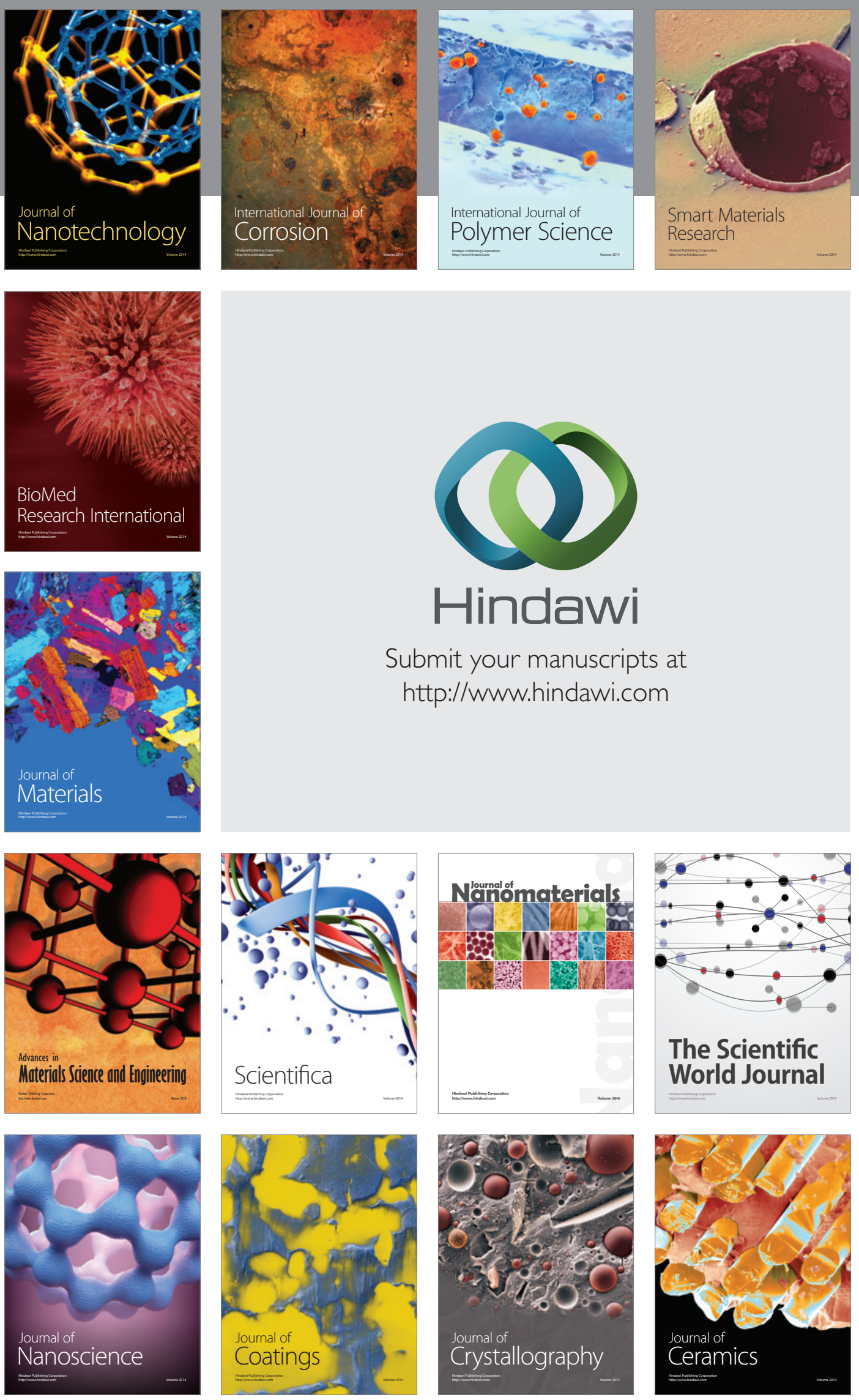

The Scientific World Journal

Submit your manuscripts at

http://www.hindawi.com

\section{World Journal}

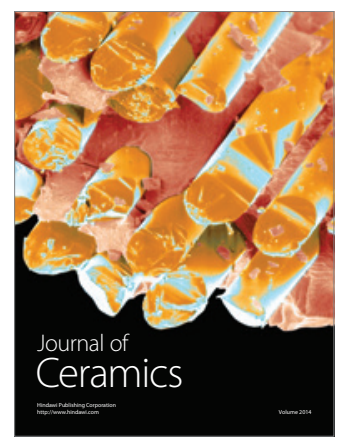

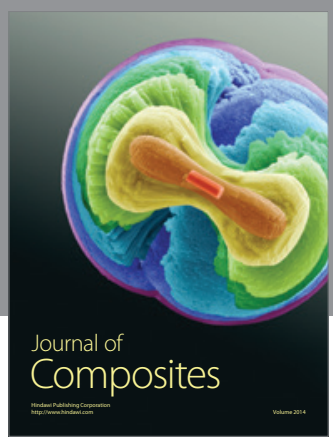
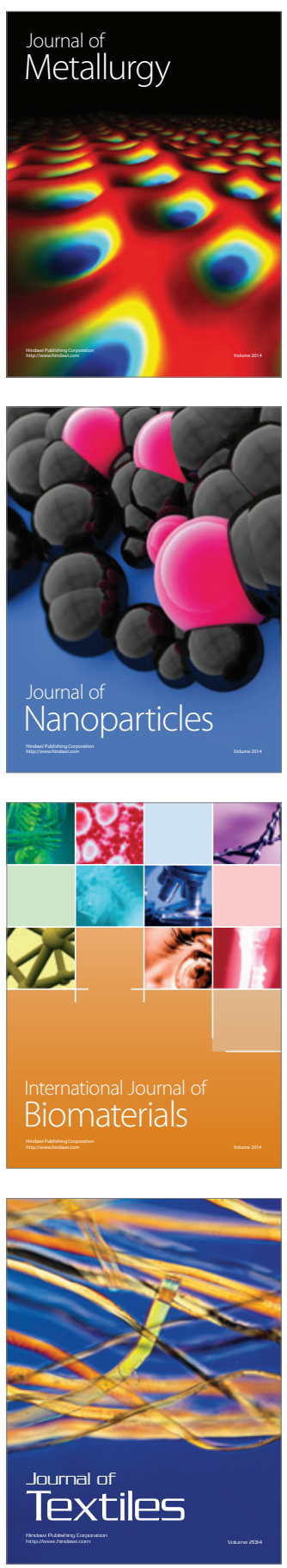